\title{
PA-127 INTERRUPTED BANCROFTIAN FILARIASIS EXPOSURE RATES IN CHILDREN AFTER TWELVE ROUNDS OF MASS DRUG ADMINISTRATION AND USE OF LONG-LASTING INSECTICIDAL NETS IN RUFIJI DISTRICT, TANZANIA
}

Clarer Jones, ${ }^{1}$ Billy Ngasala, ${ }^{1}$ Mwelecele Malecela2. 'MUHAS, Tanzania; ${ }^{2}$ NIMR, Tanzania

\subsection{6/bmjgh-2016-000260.154}

Background Tanzania started implementing the WHO strategy of mass drug administration (MDA) with ivermectin and albendazole to eliminate lymphatic filariasis (LF) in Rufiji District, which had a baseline prevalence of $49 \%$ in 2000. This study was conducted in April 2015, six months after the latest MDA to establish the impact of MDA and utilisation of long-lasting insecticidal nets on the exposure rates of LF among standard-one children born within the implementation period of the LF elimination programme after 12 rounds.

Methods A cross-sectional study for LF circulating filarial antigen (CFA) was performed in 5 primary schools from 5 different villages. A total of 659 standard-one pupils aged 6-9 years were recruited and screened for CFA using immunochromatographic test cards (ICT). Prior to blood sample collection, children were interviewed on their participation in the MDA. A finger prick whole blood sample $(100 \mu \mathrm{l})$ drawn from each child was applied to ICT. Results were read after ten minutes for the presence of CFA. Also, the study involved 868 heads of household who were interviewed on their participation in MDA and utilisation of long-lasting insecticidal nets (LLINs).

Results The ICT results were negative for CFA and suggest that there has been an interruption of exposure of children to LF transmission in the study area. More than half of the screened children (54.3\%) participated in 2014 MDA round. Household surveyed MDA coverage was $57.4 \%$ for the 2014 MDA, below the minimum effective coverage recommended by WHO. Majority $(92.5 \%)$ of households possessed and utilised LLINs. Of those who did not take the drugs in the last round, $88.7 \%$ possessed and utilised LLINs suggesting its synergistic effect with ivermectin and albendazole on LF transmission.

Conclusions Additional MDA rounds and utilisation of LLINs in areas of high-baseline prevalence may result in considerable decreased lymphatic filariasis infection transmission. 